\title{
Liraglutide as an Adjunct Agent to Manage Excessive Weight Regain Two Years Post Bariatric Surgery
}

P-0478

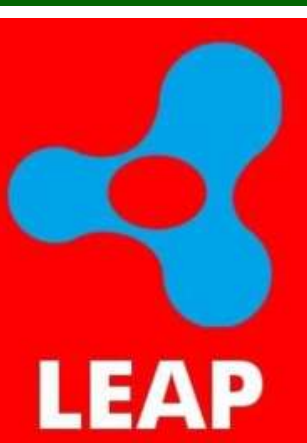

Authors: Shehla Shaikh ${ }^{1,2}$, Carlyne Remedios $^{2}$, Salomi Shaikh ${ }^{3}$,

Institution: 1. Saifee Hospital, Mumbai. 2. Dr Muffi Digestive Health Institute, Mumbai. 3. D.Y.Patil Medical College, Mumbai
IDF

2019

\section{Background}

- Bariatric surgery is an effective management modality for patients with severe or moderate obesity

- However, post-operatively weight regain due to multifactorial factors occurs in some patients, which is an important unaddressed medical concern

- There are limited evidence-based approaches available for managing weight gain in patients who have undergone bariatric surgery.

Aims

- We evaluated the clinical efficacy of GLP-1 agonist, liraglutide for managing the weight regain after at least two years of bariatric surgery

\section{Methods}

- We conducted a retrospective analysis to identify the patients who regained weight after two years after undergoing bariatric surgery from January 2016 till June 2019

- The patients were adherent to the structured lifestyle management protocols

- We extracted the key demographic data, evaluated the weight loss - post bariatric surgery and after initiation of liraglutide

- Descriptive statistics, t-test and ANOVA were used for the analysis

\section{Results}

- We evaluated the results across 11 patients (8 females and 3 males) with a mean age of 45 years (minimum 26,

maximum 64) and mean BMI $37 \mathrm{~kg} / \mathrm{m} 2$ (minimum 29, maximum 47), who underwent a varied variety of bariatric surgery procedures

- The mean weight at the time of surgery was $123 \mathrm{~kg}$ (minimum 95, maximum 159, SD $\pm 22,95 \%$ CI 109 to 138)

- The mean lowest weight achieved post-surgery was $88 \mathrm{~kg}$ (minimum 62, maximum 115, SD $\pm 16,95 \%$ CI 77 to 99), with a significant decrease in the mean weight of $35 \mathrm{~kg}$ ( $p<0.0003$ ), a decrease of $28 \%$ over the baseline.

- The mean weight at which liraglutide was initiated was 99 $\mathrm{kg}$ (minimum 80 , maximum $135, \mathrm{SD} \pm 17,95 \% \mathrm{CI} 88$ to 110) with the mean weight regained was $11 \mathrm{~kg}$ (minimum 3.5 , maximum $20, \mathrm{SD} \pm 6,95 \% \mathrm{CI} 6.8$ to 15$), 13 \%$ regain in weight over two years.

- The mean weight (99 kg) at initiation of liraglutide $0.6 \mathrm{mg}$, decreased to mean weight $(97 \mathrm{~kg}$ ) at 1st week, followed by $1.2 \mathrm{mg}$ liraglutide leading to mean weight of $96 \mathrm{~kg}$ which was stable at $95 \mathrm{~kg}$ in the subsequent follow up, with the weekly up-titration of the dose of liraglutide to $1.8 \mathrm{mg}$ and $2.4 \mathrm{mg}$

Copyright (C) 2019

Dr Shehla Shaikh drshehlas@gmail.com
- The mean decrease in the weight one month was $3.4 \mathrm{~kg}$ (3.5\%) (minimum 0.4, maximum 5.5; $\mathrm{p}=0.98 \mathrm{NS}$ ).

- None of the patients gained weight after initiation of liraglutide

Fig 1: Weight at time of Surgery Fig 4: Lowest weight

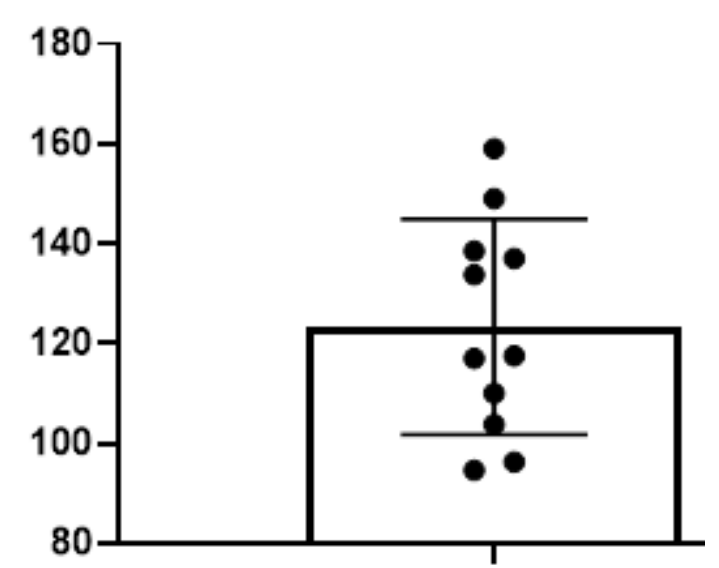
achieved after surgery

Fig 2: Weight at Initiation of Liraglutide Fig 5: Weight regained

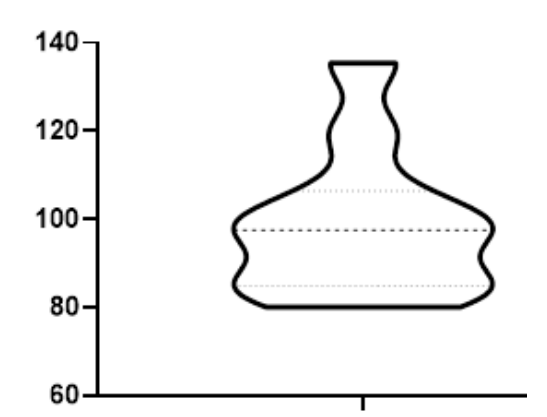

Fig 3: Age

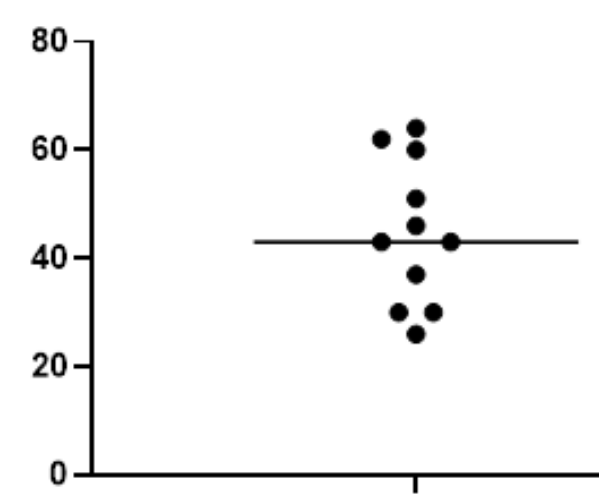

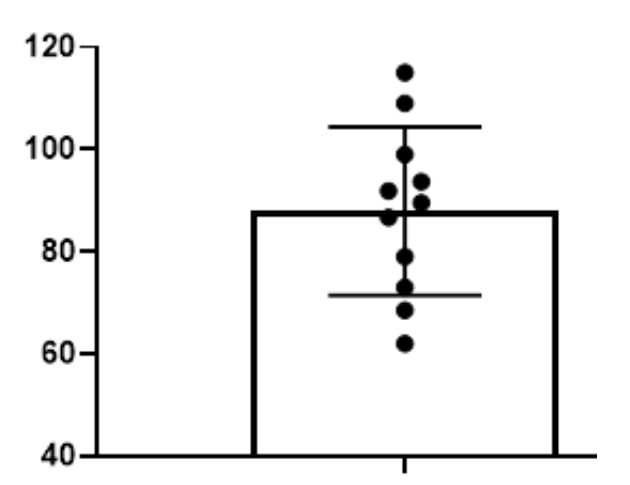

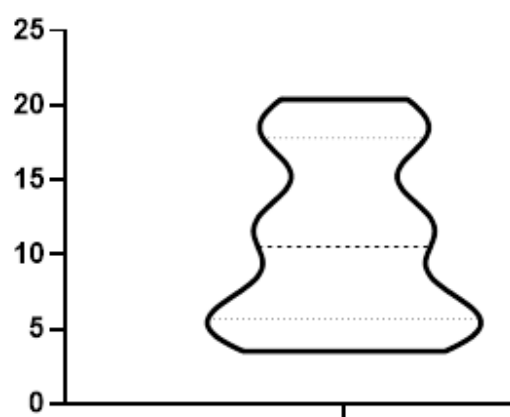

Fig 6: BM

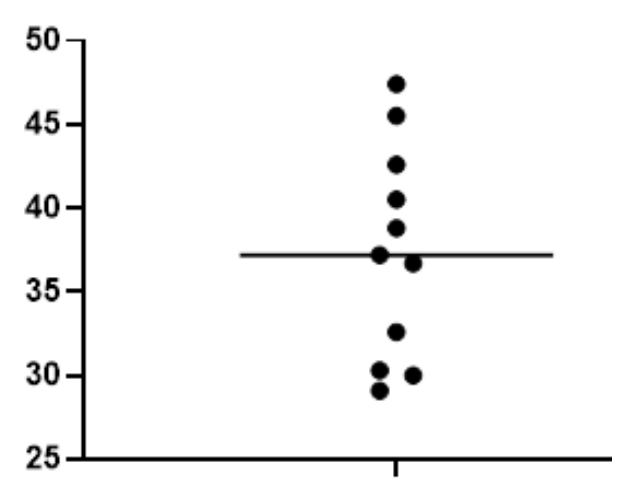

Fig 7: Values of weight on liraglutide

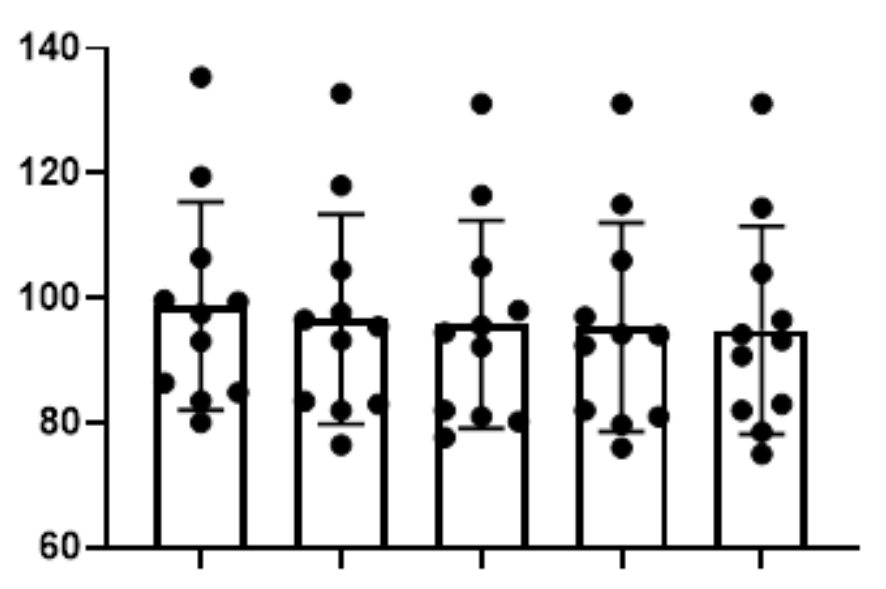

Discussion

- The initial results suggest that liraglutide as a weight-loss pharmacotherapeutic agent in routine clinical care is a useful adjunct to the bariatric surgery in patients with weight regain, regardless of the surgery type.

- The ongoing long term follow up will further provide the evidence for the benefits of liraglutide in these patients 